\title{
Interleukin-13 reduces cardiac injury and prevents heart dysfunction in viral myocarditis via enhanced M2 macrophage polarization
}

\author{
Honghui Yang ${ }^{1}$, Yan Chen ${ }^{1}$ and Chuanyu Gao ${ }^{1}$ \\ ${ }^{1}$ Department of Cardiology, Zhengzhou University People's Hospital, Zhengzhou 450001, China \\ Correspondence to: Honghui Yang, email: yhh6232@yeah.net \\ Keywords: IL-13, viral myocarditis, macrophage polarization
}

Received: July 02, $2017 \quad$ Accepted: July 26, $2017 \quad$ Published: August 10, 2017

Copyright: Yang et al. This is an open-access article distributed under the terms of the Creative Commons Attribution License 3.0 (CC BY 3.0), which permits unrestricted use, distribution, and reproduction in any medium, provided the original author and source are credited.

\begin{abstract}
Viral myocarditis is one of the major causes of congestive heart failure and dilated cardiomyopathy. Recent reports have demonstrated an essential role of cytokines, like interleukin-13 (IL-13), in the pathogenesis of viral myocarditis, while the underlying mechanisms remain poorly defined. Here, using a coxsackie virus B3 (CVB3)-infection model in BALB/C mice, we showed that IL-13 protected mouse heart function in viral myocarditis, seemingly through reduction in $\mathrm{T}$ lymphocyte immunity and induction of M2 macrophage polarization. Adoptive transfer to M2 macrophages mimicked the effects of IL-13 on protection from myocarditis, suggesting that the effects of IL-13 may be primarily through regulation of macrophage polarization. Together, our data suggest that application of IL-13 treatment may reduce cardiac Injury and protect heart function in viral myocarditis via enhanced M2 macrophage polarization.
\end{abstract}

\section{INTRODUCTION}

Some viruses, like coxsackie virus and hepatitis $\mathrm{C}$ virus, are known to cause viral myocarditis, as a cause for the subsequent development of dilated cardiomyopathy [1]. Recent reports have demonstrated an essential role of cytokines in the pathogenesis of viral myocarditis [2-4]. First of all, high tumor necrosis factor (TNF)-alpha, Interleukin (IL)-1-alpha and IL-1-beta were detected in the plasma of myocarditis patients with congestive heart failure [5, 6]. Moreover, granulocyte colony-stimulating factor was often upregulated in myocarditis, suggesting activation of macrophages [7-10].

These evidence of involvement of cytokines in the pathogenesis of myocarditis thus led to the approaches of using inhibitory cytokines and cytokine suppression, e.g. IL-1 receptor antagonist or IL-10 [11], or suppressing IL-4 [12], in a cytokine therapy. For example, blockade of IL-4 partially suppresses the development of experimental viral myocarditis in $\mathrm{A} / \mathrm{J}$ mice, suggesting involvement of Th2 cytokines in the pathology of viral myocarditis [12]. Interestingly, IL-13 knockout (KO) mice was later found to develop enhanced experimental viral myocarditis in $\mathrm{BALB} / \mathrm{C}$ mice [13]. As a pleiotropic cytokine produced by many hematopoietic cell types, IL-13 does not use the classical IL-4 receptor, but an alternative IL-4 receptor, consisting of IL-4R-alpha and IL-13 receptor alpha1 subunit [14]. Moreover, IL-13 has a decoy receptor named IL-13R-alpha2 for exerting antagonistic functions [14]. These characteristics determine a distinct function of IL13 from IL-4 during immune responses.

Macrophages are capable of differentiating into different subtypes with a range of function in respond to various environmental cues, which is called macrophage polarization [15-17]. Macrophage M1 and M2-type responses exert the opposing activities of either killing or repairing, by which such polarized responses correspondingly induce Th1- or Th2-like responses in macrophages, respectively [15]. The typical characteristics of M1 macrophages include predominant antigen presentation, high production of IL-12 and IL-23, and high production of nitric oxide (NO) and reactive oxygen species. On the other hand, M2-type responses are mainly induced by IL-4, IL-10, or IL-13 [15]. M2 macrophages are typically characterized by the upregulation of Dectin-1, mannose receptor (CD301), scavenger receptor, CD163 and CD206. Moreover, M2 likely upregulates arginase, compared to M1 macrophages 
[15]. While M1 macrophages enhance Th1 response, M2 macrophages augment $\mathrm{Th} 2$ response, tissue remodeling and immune tolerance [15]. A coordination of a number of inflammatory modulators, signaling molecules, and transcription factors controls macrophage polarization. Activation of canonical IRF/STAT1 signaling by interferon-gamma and toll-like receptor signaling will polarize macrophages toward the M1 phenotype, while activation of IRF/STAT6 signaling by IL-4 and IL-13 will polarize macrophages toward the M2 phenotype [15]. Although these cytokines play a critical role in macrophage differentiation and polarization, its effects on viral myocarditis through macrophage polarization have not systemically studied.

In the current study, we investigated the effects of application of IL-13 treatment on cardiac Injury and protection of heart function in viral myocarditis as well as the involvement of macrophage polarization in the process.

\section{RESULTS}

\section{IL-13 protects mouse heart function in coxsackie virus B3 (CVB3)-induce myocarditis}

We gave coxsackie virus B3 (CVB3) to BALB/C mice, and analyzed development of viral myocarditis 5 weeks after infection. To evaluate the effects of IL13 on myocarditis development, at the time of viral injection, we injected some mice with recombinant mouse IL-13 every other day for 3 times. Mice were divided into 3 groups: Group 1, mice were injected with saline as a control for CVB3 injection (Sham); Group 2 , mice were injected with CVB3, but for control of IL13 administration, saline of same dose and frequency was given (CVB3); Group 3: mice were injected with CVB3 and IL-13 (CVB3+IL-13) (Figure 1A). At week 5 , the infection of the mouse heart was determined, showing that CVB3 significantly increased heart inflammation, which was significantly attenuated by IL13 injection, shown by quantification (Figure 1B), and by representative images (Figure 1C). The heart function of the mice from 3 groups was assessed at analysis. We found that CVB3 treatment significantly increased the left ventricular end diastolic dimension (LVEDD; Figure 1D) and left ventricular end systolic dimension (LVESD; Figure 1E) measured from a frozen M-mode tracing, and significantly reduced the percentage of Fractional shortening ( $\%$ Fractional shortening; Figure $1 \mathrm{~F})$ and the percentage of Ejection fraction (\% Ejection fraction; Figure $1 \mathrm{G}$ ). Interestingly, IL-13 administration attenuated the detrimental effects of OVB3 on these parameters (Figure 1D-1G), suggesting that IL-13 may protect mouse heart function in CVB3-induce myocarditis.

\section{IL-13 reduces $T$ lymphocyte immunity and induces $\mathrm{M} 2$ macrophage polarization}

Next, we examined the underlying mechanisms. Since $\mathrm{T}$ lymphocytes are key players in the development of viral myocarditis, and since IL-13 is believed to be predominantly macrophage regulator, we used flow cytometry to examine these 2 populations in the mouse heart. First, we analyzed the percentage of $\mathrm{CD} 3+$ lymphocytes in mouse heart. We found that CVB3 significantly increased CD3+ lymphocytes in the mouse heart than control mice (Sham), but the increases in CD3+ cells was significantly attenuated by IL-13 injection, shown by representative flow charts (Figure 1A), and by quantification (Figure 1B). Moreover, CVB3 significantly increased $\mathrm{F} 4 / 80+$ macrophages in the mouse heart than control mice (Sham; Figure 1C-D). Interestingly, although IL-13 did not alter CD163-F4/80+ M1 macrophages in the CVB3-treated mouse heart, IL-13 significantly increased the CD163+F4/80+ M2 macrophages in the CVB3treated mouse heart, shown by quantification (Figure 1C), and by representative flow charts (Figure 1D). Thus, IL-13 reduces T lymphocyte immunity and induces M2 macrophage polarization in CVB3-induce myocarditis.

\section{Preparation of IL-13-induced M2 macrophages for transplantation}

In order to evaluate the role of M2 macrophages in the IL-13-attenuated CVB3-myocarditis, we isolated mouse macrophages from bone marrow of age-matched, isogeneic mice. IL-13 was given to these macrophages to induce a M2-macrophage polarization, which mimicked the in vivo model (Figure 3A). We found that IL-13 treatment significantly induced CD163 expression in these macrophages, suggesting M2 M2-macrophage polarization (Figure 3B). In order to confirm the change of phenotype, mRNA was isolated from the treated cells and subjected to a RT-qPCR analysis for macrophage subtype-specific genes. We found that IL-13-treated cells significantly reduced iNOS expression, and significantly increased Arginase and Declin-1 expression (Figure 3C), suggesting a M2-like polarization. These cells were thus readily used for adoptive transplantation in a gain-offunction experiment.

\section{Adoptive transfer of M2 macrophages mimics the effects of IL-13 in CVB3-myocardits}

The IL-13-treated macrophages were used for adoptive transplantation in CVB3-myocardits. At the time of viral injection, $10^{6} \mathrm{IL}-13$-treated macrophages were i.p. injected to some mice every other day for 3 times. The first injection was done at the time of CVB3 injection. CVB3-treated mice were divided into 2 groups. Group 1, 
mice were injected with CVB3, but for control of adoptive transfer of M2 macrophages, saline of same dose and frequency was given (CVB3); Group 3: mice were injected with $\mathrm{CVB} 3$ and received adoptive transfer of M2 macrophages $(\mathrm{CVB} 3+\mathrm{M} 2 \mathrm{M} \varphi)$ (Figure 4A). At week 5, the infection of the mouse heart was determined, showing that CVB3 significantly increased heart inflammation, which was significantly attenuated by adoptive transfer of M2
A

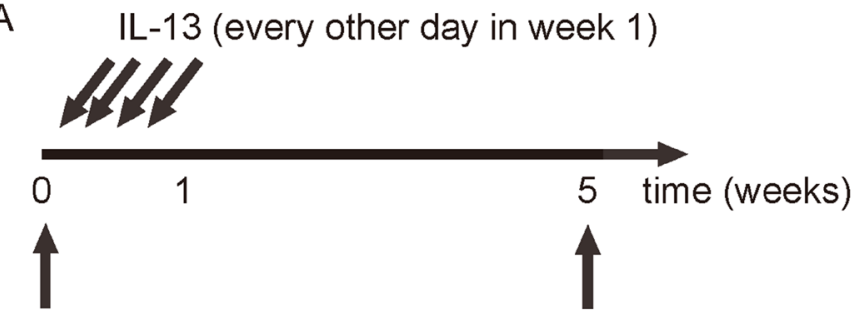

CVB3

analysis

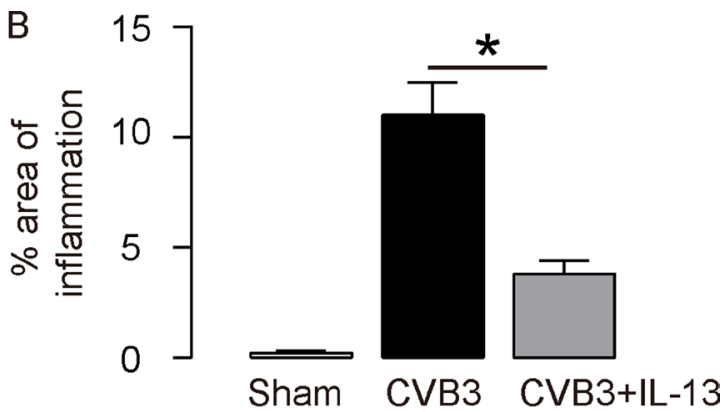

C

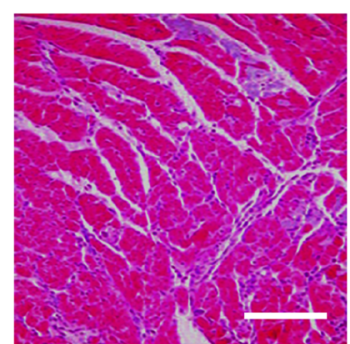

Sham

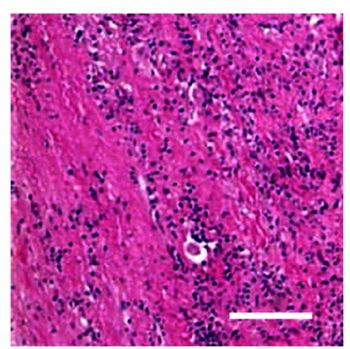

CVB3

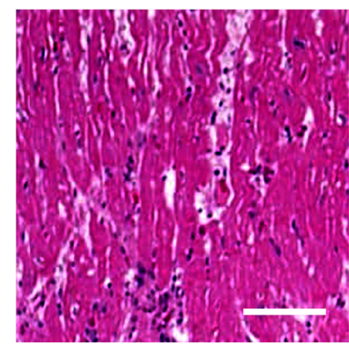

CVB3+IL-13
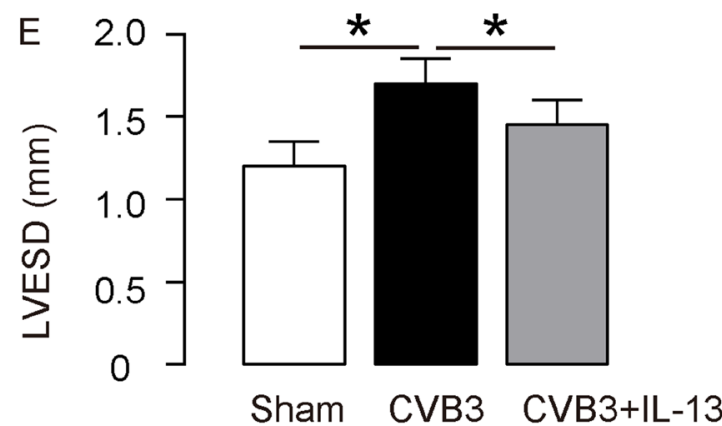

Sham CVB3 CVB3+IL-13
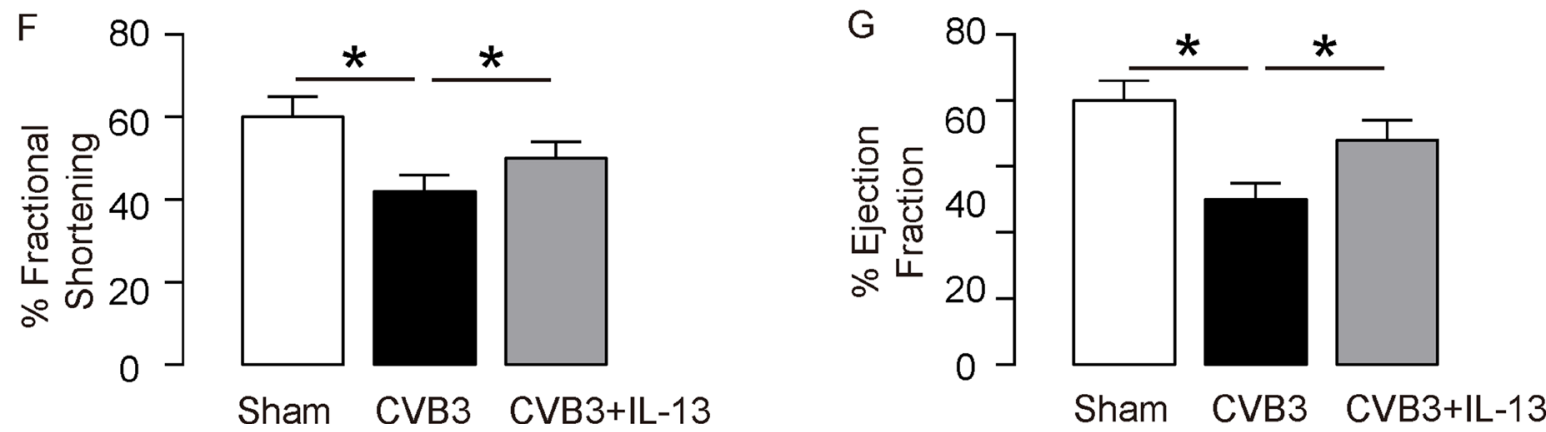

Figure 1: IL-13 protects mouse heart function in coxsackie virus B3 (CVB3)-induce myocarditis. (A) We gave CVB3 to $\mathrm{BALB} / \mathrm{C}$ mice, and analyzed development of viral myocarditis 5 weeks after infection. To evaluate the effects of IL-13 on myocarditis development, at the time of viral injection, we injected some mice with recombinant mouse IL-13 every other day for 3 times. Mice were divided into 3 groups: Group 1, mice were injected with saline as a control for CVB3 injection (Sham); Group 2, mice were injected with CVB3, but for control of IL-13 administration, saline of same dose and frequency was given (CVB3); Group 3: mice were injected with CVB3 and IL-13 (CVB3+IL-13). (B-C) The infection severity of the mouse heart was determined at week 5, showing by quantification (B), and by representative images (C). (D-G) Assessment of mouse heart function at week 5. (D) LVEDD. (E) LVESD. (F) \% Fractional shortening. (G) \% Ejection fraction. $N=10 .{ }^{*} p<0.05$. Scale bars are $50 \mu \mathrm{m}$. 
macrophages, shown by quantification (Figure 4B), and by representative images (Figure 4C). The heart function of the mice from 2 groups was assessed at analysis. We found that CVB3 treatment significantly increased the LVEDD (Figure 4D) and LVESD (Figure 1E), and significantly reduced the $\%$ Fractional shortening (Figure $1 \mathrm{~F}$ ) and the $\%$ Ejection fraction (Figure 1G). Like IL-13, adoptive transfer of M2 macrophages attenuated the detrimental effects of OVB3 on these parameters (Figure 4D-4G), suggesting that the effects of IL-13 on CVB3-myocarditis may be primarily through induction of M2 macrophage polarization.

\section{DISCUSSION}

Recent reports suggest importance of cytokines in the pathology of viral myocarditis [2-4]. Moreover, approaches of inhibitory cytokine application and suppression of certain cytokine have been used in animal model for viral myocarditis [11, 12]. Unlike IL-4 [12], IL13 knockout $(\mathrm{KO})$ mice develop enhanced experimental viral myocarditis in BALB/C mice [13], but the effects of IL-13 treatment have not been examined.

The target cells of IL-4 and IL-13 are different. Although they may share IL-4R-alpha subunit, IL-13

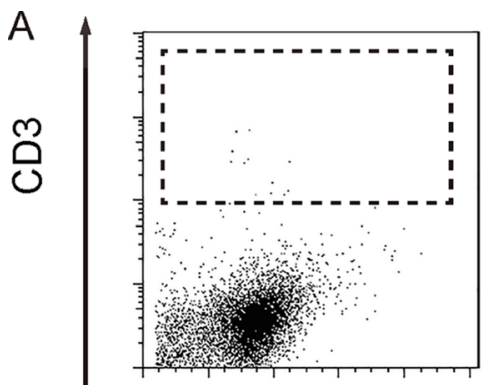

Sham

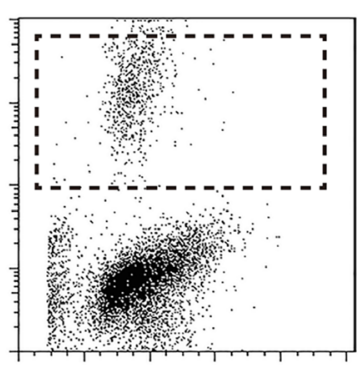

CVB3

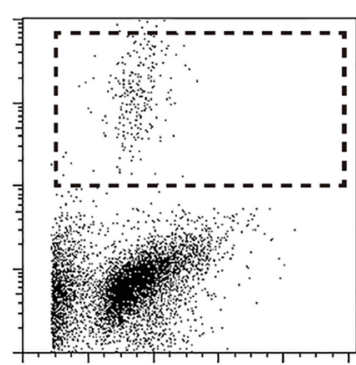

CVB3+IL-13

\section{FSC}
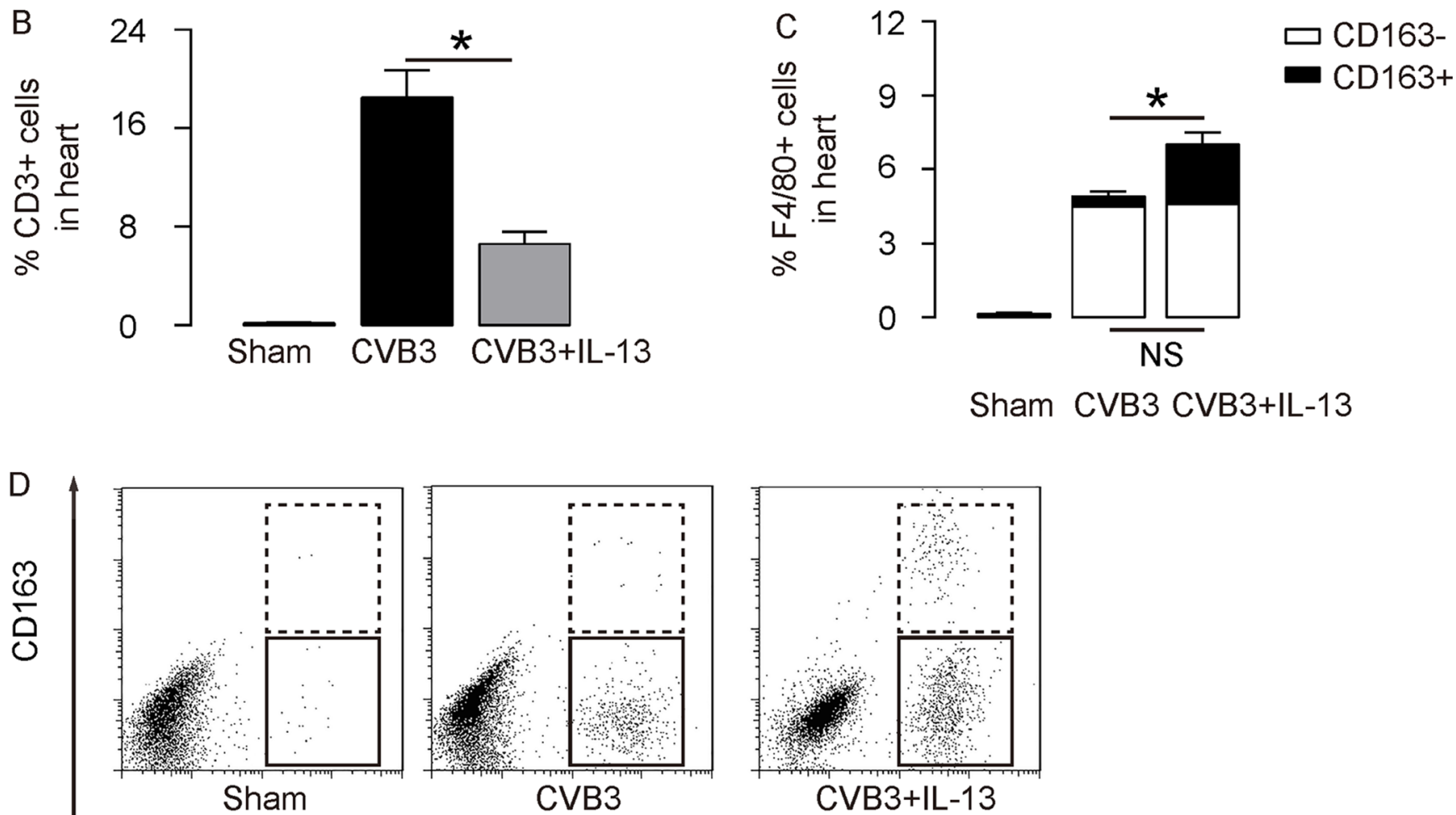

CVB3

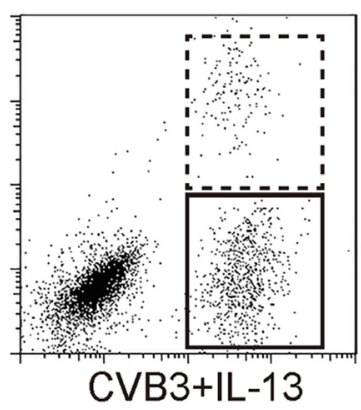

\section{F4/80}

Figure 2: IL-13 reduces T lymphocyte immunity and induces M2 macrophage polarization. (A-B) The percentage of CD3+ lymphocytes in mouse heart was assessed by flow cytometry, shown by representative flow charts (A), and by quantification (B). (C-D) The percentage of CD163-F4/80+ M1 macrophages and CD163+F4/80+ M2 macrophages in the mouse heart were assessed by flow cytometry, shown by quantification (C), and by representative flow charts (D). $N=10$. NS: non-significant. ${ }^{*} p<0.05$. 
processes different receptor subunits, IL-13R-alpha1 and IL-13R-alpha2 [14]. Since T cells do not express functional IL-13R-alpha1, IL-13 appeared to have no effects on $\mathrm{T}$ cells, but relatively exclusive effects on monocytes/macrophages. Hence, we believed that IL13 may be primarily effective through macrophages in the current model, which was then confirmed in our experiments.

In contrast to interferon-gamma which specifically activates M1 macrophages, IL-13 and IL-4 are wellknown to activate M2 macrophages. But when taking into concern of the specificity of target cells, modulation of IL13 , rather than IL-4, should have relative clearer effects on immunity, which results in the complete opposite effects of them on viral myocarditis $[12,13]$.

Compared to M1 macrophages, the role of M2 macrophages during infection and tissue injury/repair appears to be mainly suppression of immune responses and enhancement of tissue repair [15].

In our experiments, we found that IL-13 increased M2 macrophages, but did not alter M1 macrophage number. This effect resulted in alteration of the ratio of M1 versus M2 macrophages, and the alteration of growth factor secretion from macrophages, which may subsequently change the differentiation and function of $\mathrm{T}$ cells. Indeed, we found that the total $\mathrm{T}$ cells were decreased by IL-13, as a direct cause for the attenuation of the viral myocarditis. On the other hand, the unaltered number of M1 macrophages by IL-13 allowed us to use adoptive M2 macrophage transfer, rather than combination of adoptive M2 macrophage transfer and M1 macrophage elimination method, in a gain-of-function approach.
The data from adoptive M2 macrophage transfer well supported the unique effects of IL-13 on macrophages, and confirmed the effects of IL-13 on heart function protection in primarily through M2 macrophage polarization.

Recently, macrophage polarization has been found out to play very critical roles in numerous diseases, e.g. tumor formation [18-21], tissue repair [22-24], and cell proliferation [20, 25-29]. Compared to adoptive transfer of M2 macrophages, in vivo polarization of macrophages may be a more powerful and clinic-applicable method, since macrophage polarization in vivo not only increases M2 macrophages, but also reduces M1 macrophages, resulting in two beneficial effects of further increases in production of trophic growth factors and removal of the detrimental effects from M1 macrophages [25, 30-32]. Recent studies have reported these approaches, e.g. used of TIPE2 [33-36]. Hence, these strategies may be tested in future studies.

To summarize, our study contributes to a clear demonstration of an IL-13-mediated protective pathway in the pathogenesis of viral myocarditis. Further dissection of the detailed cell-cell crosstalk and the underlying molecular mechanism may improve our understanding of the viral myocarditis in humans.

\section{MATERIALS AND METHODS}

\section{Protocol approval and mouse housing}

All methods and protocols involving mouse manipulations were approved by the Animal Care and Use Committee of Zhengzhou University People's Hospital.
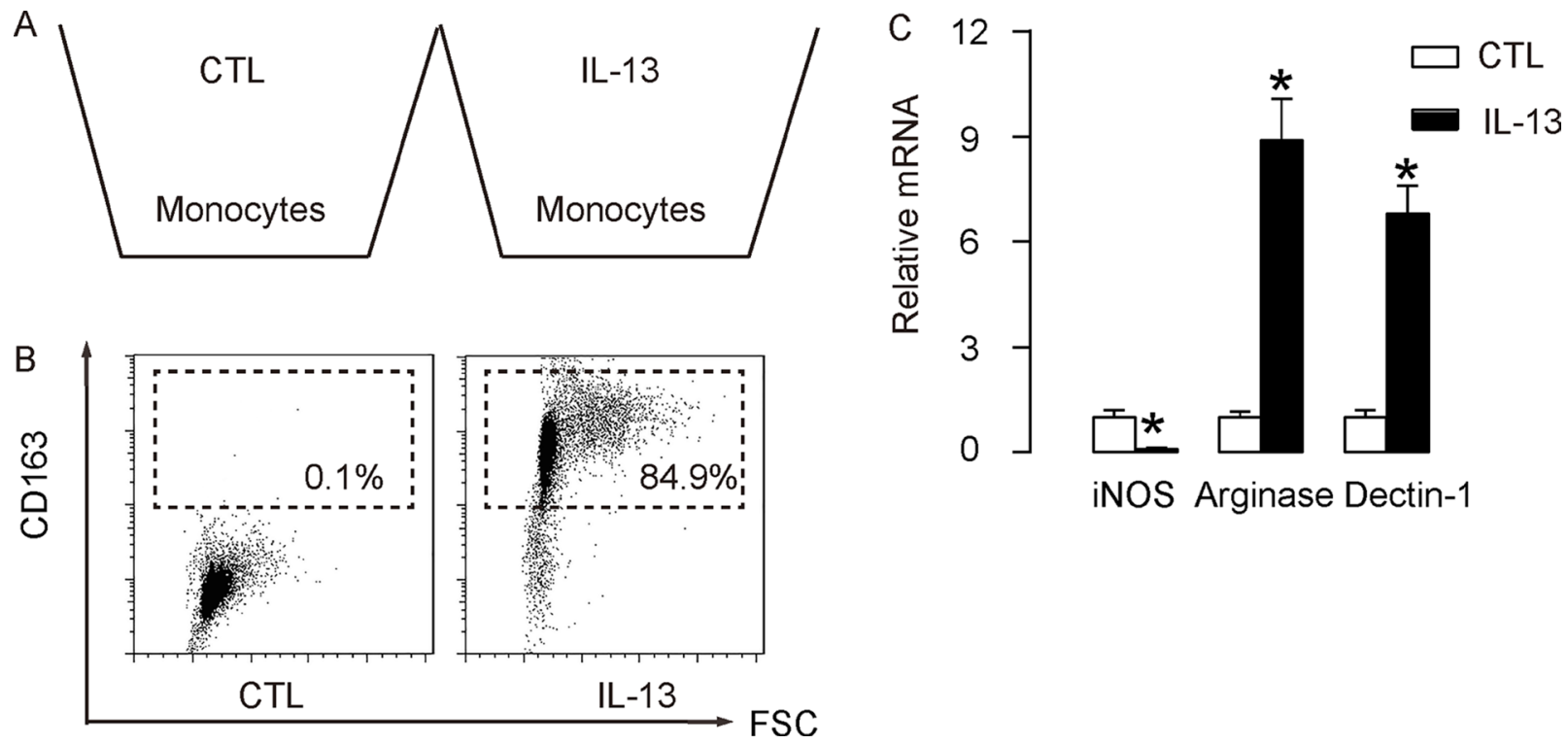

Figure 3: Preparation of IL-13-induced M2 macrophages for transplantation. (A) Mouse macrophages were isolated from bone marrow of age-matched, isogeneic mice. IL-13 was given to these macrophages to induce a M2-macrophage polarization, which mimicked the in vivo model. (B) Representative flow chart for analysis of CD163 expression in IL-13-treated macrophages, compared to control (CTL). (C) RT-qPCR for iNOS, Arginase and Declin-1 expression in IL-13-treated macrophages, compared to CTL. $N=5$. ${ }^{*} p<0.05 . \mathrm{M} \varphi$ : macrophages. 
A

$\mathrm{M} 2 \mathrm{M} \varphi$ (every other day in week 1)

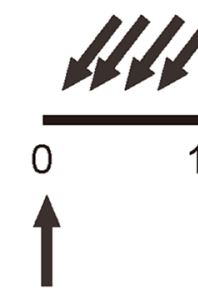

CVB3

C

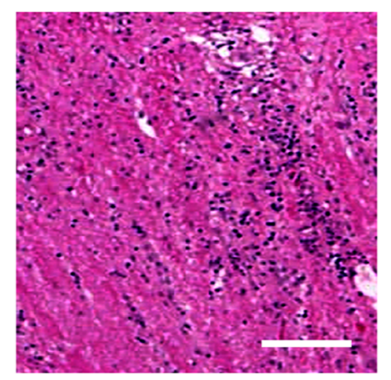

CVB3

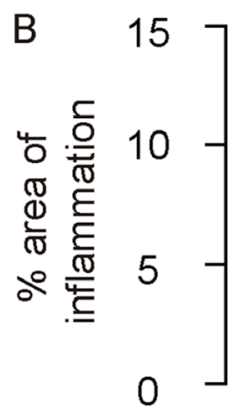

analysis

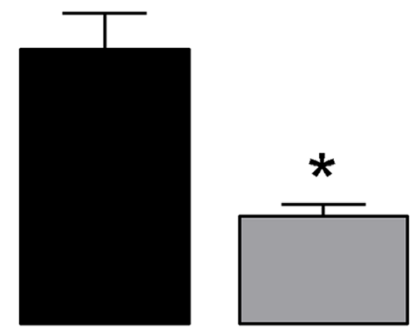

CVB3 CVB3+M2M $\varphi$

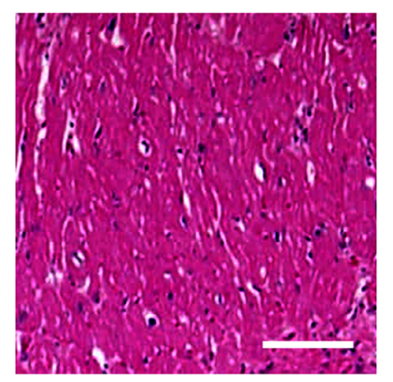

$\mathrm{CVB} 3+\mathrm{M} 2 \mathrm{M} \varphi$
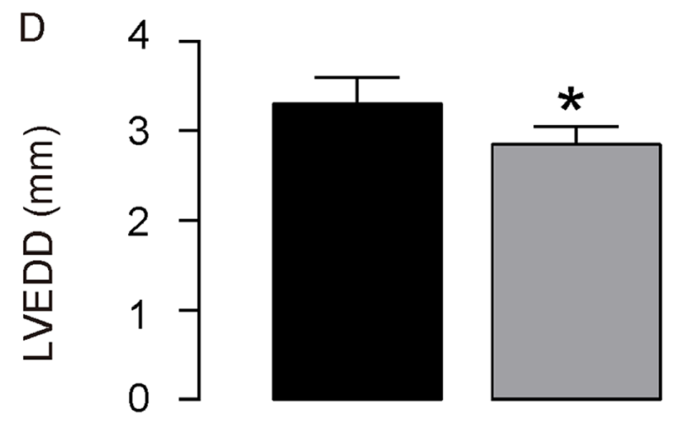

CVB3 CVB3+M2M $\varphi$

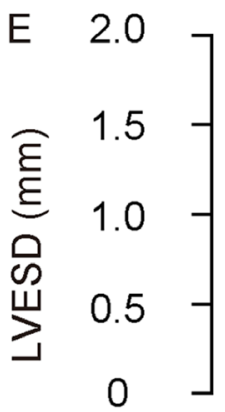

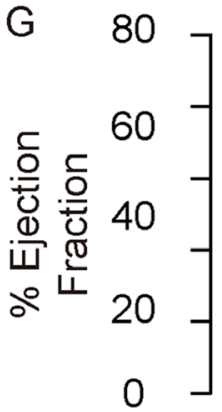

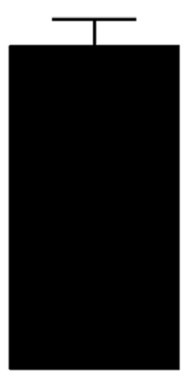

CVB3 CVB3+M2M $\varphi$
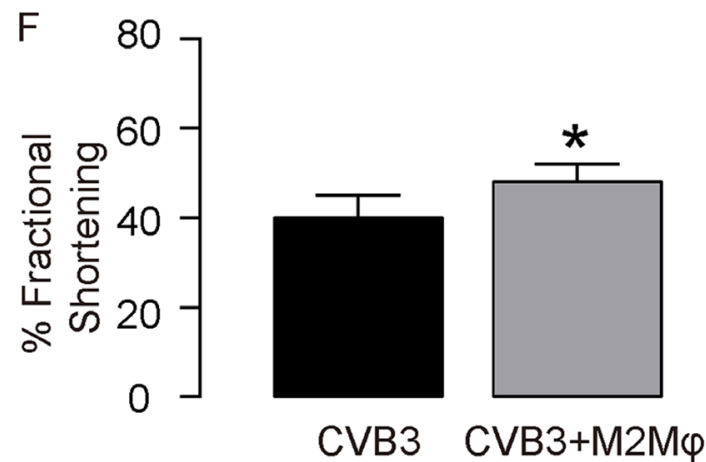

CVB3

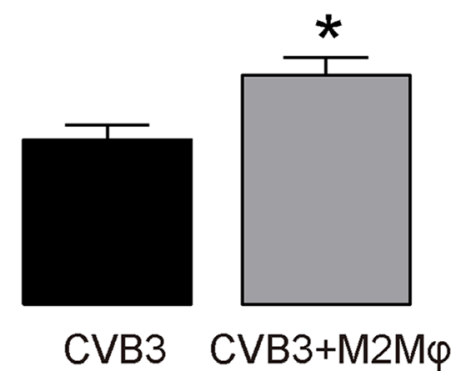

Figure 4: Adoptive transfer of M2 macrophages mimics the effects of IL-13 in CVB3-myocardits. (A) Experimental design: At the time of viral injection, $10^{6} \mathrm{IL}-13$-treated macrophages were i.p. injected to some mice every other day for 3 times. The first injection was done at the time of CVB3 injection. CVB3-treated mice were divided into 2 groups. Group 1, mice were injected with CVB3, but for control of adoptive transfer of M2 macrophages, saline of same dose and frequency was given (CVB3); Group 3: mice were injected with CVB3 and received adoptive transfer of $\mathrm{M} 2$ macrophages (CVB3+M2M $\varphi)$. (B-C) The infection severity of the mouse heart was determined at week 5, showing by quantification (B), and by representative images (C). (D-G) Assessment of mouse heart function at week 5. (D) LVEDD. (E) LVESD. (F) \% Fractional shortening. (G) \% Ejection fraction. $N=10 .{ }^{*} p<0.05$. Scale bars are $50 \mu$ m. M $\varphi$ : macrophages. 
Male BALB/C mice of 12 weeks of age were purchased from Shanghai Laboratory Animal Center (Shanghai, China), and maintained in the Zhengzhou University People's Hospital conventional animal facility.

\section{Coxsackie virus B3 (CVB3)-induced viral myocarditis model in mice}

For developing CVB3-induced myocarditis, Male $\mathrm{BALB} / \mathrm{C}$ mice of 12 weeks of age were inoculated intraperitoneally with 800 plaque-forming units of a heartpassaged stock of CVB3 (Nancy strain, American Type Culture Collection, ATCC, Rockville, MD, USA), diluted in sterile phosphate-buffered saline (PBS). Recombinant mouse IL-13 (R\&D Systems, Los Angeles, CA, USA) was given subcutaneously at a dose of $5 \mu \mathrm{g} /$ mouse every other day in the first week after viral infection. The first injection was done at the time of viral injection.

\section{Isolation, culture and differentiation of mouse macrophages}

Mouse macrophages were isolated from bone marrow, as described before [37]. Marrow from male, 12 week-old BALB/C mice was flushed out with PBS containing $20 \mathrm{mmol} / 1$ Tris and $100 \mathrm{mmol} / 1 \mathrm{NaCl}(\mathrm{pH} 7.5)$ through a 23-gauge needle. Cells were pre-treated with FITC-conjugated F4/80 antibody (Becton-Dickinson Biosciences, San Jose, CA, USA) and then sorted for F4/80+ cells by flow cytometry. Purified F4/80+ macrophages were cultured in Dulbecco's Modified Eagle Medium/F12 (DMEM/F12; Invitrogen, St. Louis, MO, USA) suppled with $10 \mathrm{mmol} / \mathrm{l}$ L-glutamine, 100U/ $\mathrm{ml}$ penicillin, $100 \mu \mathrm{g} / \mathrm{ml}$ streptomycin and $100 \mathrm{U} / \mathrm{ml}$ recombinant M-CSF (R\&D Systems). For induction of macrophage polarization in vitro by IL-13, cultured macrophages were treated with recombinant IL-13 (R\&D Systems, cat. no. 413-M) at a concentration of $20 \mathrm{U} / \mathrm{ml}$ and incubate overnight.

\section{Fluorescence-activated cell sorting (FACS) for leukocytes/macrophage (subtypes)}

Heart tissue was minced into small pieces of $1 \mathrm{~mm}$ in diameter, and then subjected to $0.25 \%$ Trypsine solution (Invitrogen) for 45 minutes, filtered through $45 \mu \mathrm{m}$ filter, washed 3 times with vehicle solution, and re-suspended in PBS. Cultured cells were dissociated and then analyzed directly. These single cell fractions were incubated with either PE-conjugated anti-CD3 antibody, or FITCconjugated anti-F4/80 antibody and/or APC-conjugated anti-CD163 antibody (Becton-Dickinson Biosciences), and then sorted for leukocytes, macrophage (M1 or M2), correspondingly. Data were analyzed and quantified using Flowjo software (Flowjo LLC, Ashland, OR, USA).

\section{Determination of histopathological changes}

Mice were evaluated for the development of CVB3induced viral myocarditis 5 weeks after infection. Mouse Heart tissue was fixed in $10 \%$ phosphate-buffered formalin, after which paraffin embedding was performed. Longitudinal sections of $5 \mu \mathrm{m}$-thickness were prepared for hematoxylin and eosin staining. The severity of the CVB3-induced viral myocarditis was assessed as the percentage of the heart section with inflammation compared with the overall heart section area, determined by 2 independent researchers who scored the slides separately in a blinded manner.

\section{Echocardiography}

Trans-thoracic echocardiography was performed using the VisualSonic Vevo 660 imaging system equipped with a $40 \mathrm{MHz}$ transducer (VisualSonics Inc., Toronto, Canada). Ultrasonic transmission gel (Parker Laboratories, Fairfield, NJ) was applied to shaved left hemi-thorax. The mouse heart was imaged in the two-dimensional mode in the parasternal short axis view. The left ventricular end diastolic dimension (LVEDD) and left ventricular end systolic dimension (LVESD) were measured from a frozen M-mode tracing. Percentage of Fractional shortening (\% Fractional shortening) is the percent change in left ventricular cavity dimensions. Percentage of Ejection fraction (\% Ejection fraction) is the percentage of stroke volume to the end diastolic left ventricular volume.

\section{Quantitative real-time PCR (RT-qPCR)}

Total RNA were extracted using the RNeasy mini kit (Qiagen, Hilden, Germany). Complementary DNA preparation and quantitative real-time PCR (RT-qPCR) were performed, using QuantiTect SYBR Green PCR Kit (Qiagen). All primers were purchased from Qiagen. Data were collected and analyzed using $2-\Delta \Delta \mathrm{Ct}$ method. Values of genes were first normalized against housekeeping gene $\alpha$-tubulin, and then compared to the experimental controls.

\section{Statistical analysis}

All values represent the mean \pm standard deviation (SD). Statistical analysis of group differences was carried out using a one-way analysis of variance (ANOVA) test followed by the Fisher's Exact Test to compare two groups (GraphPad Software, Inc. La Jolla, CA, USA). A value of $p<0.05$ was considered statistically significant after Bonferroni correction.

\section{CONFLICTS OF INTEREST}

The authors have declared that no competing interests exist. 


\section{REFERENCES}

1. Pollack A, Kontorovich AR, Fuster V, Dec GW. Viral myocarditis - diagnosis, treatment options, and current controversies. Nat Rev Cardiol. 2015; 12:670-680.

2. Zhong $\mathrm{C}$, Wu Y, Chang $\mathrm{H}$, Liu C, Zhou L, Zou J, Qi Z. Effect of PKC inhibitor on experimental autoimmune myocarditis in lewis rats. Oncotarget. 2017; 8:5418754198. https://doi.org/10.18632/oncotarget.17018.

3. Wang D, Li T, Cui H, Zhang Y. Analysis of the Indicating Value of Cardiac Troponin I, Tumor Necrosis Factoralpha, Interleukin-18, Mir-1 and Mir-146b for Viral Myocarditis among Children. Cell Physiol Biochem. 2016; 40:1325-1333.

4. Yu M, Hu J, Zhu MX, Zhao T, Liang W, Wen S, Li HH, Long Q, Wang M, Guo HP, Cheng X, Liao YH, Yuan J. Cardiac fibroblasts recruit Th17 cells infiltration into myocardium by secreting CCL20 in CVB3-induced acute viral myocarditis. Cell Physiol Biochem. 2013; 32:1437-1450.

5. Shioi T, Matsumori A, Sasayama S. Persistent expression of cytokine in the chronic stage of viral myocarditis in mice. Circulation. 1996; 94:2930-2937.

6. Matsumori A, Yamada T, Suzuki H, Matoba Y, Sasayama S. Increased circulating cytokines in patients with myocarditis and cardiomyopathy. British heart journal. 1994; 72:561-566.

7. Hiraoka Y, Kishimoto C, Takada H, Suzaki N, Shiraki K. Colony-stimulating factors and coxackievirus B3 myocarditis in mice: macrophage colony-stimulating factor suppresses acute myocarditis with increasing interferonalpha. Am Heart J. 1995; 130:1259-1264.

8. Vasconcelos JF, Souza BS, Lins TF, Garcia LM, Kaneto CM, Sampaio GP, de Alcantara AC, Meira CS, Macambira SG, Ribeiro-dos-Santos R, Soares MB. Administration of granulocyte colony-stimulating factor induces immunomodulation, recruitment of $\mathrm{T}$ regulatory cells, reduction of myocarditis and decrease of parasite load in a mouse model of chronic Chagas disease cardiomyopathy. FASEB J. 2013; 27:4691-4702.

9. Shimada K, Okabe TA, Mikami Y, Hattori M, Fujita M, Kishimoto C. Therapy with granulocyte colony-stimulating factor in the chronic stage, but not in the acute stage, improves experimental autoimmune myocarditis in rats via nitric oxide. J Mol Cell Cardiol. 2010; 49:469-481.

10. Hiraoka Y, Kishimoto C, Takada H, Suzaki N, Shiraki K. Effects of granulocyte colony-stimulating factor upon coxsackievirus B3 myocarditis in mice. Eur Heart J. 1995; 16:1900-1906.

11. Nishio R, Matsumori A, Shioi T, Ishida H, Sasayama S. Treatment of experimental viral myocarditis with interleukin-10. Circulation. 1999; 100:1102-1108.

12. Afanasyeva M, Wang Y, Kaya Z, Park S, Zilliox MJ, Schofield BH, Hill SL, Rose NR. Experimental autoimmune myocarditis in $\mathrm{A} / \mathrm{J}$ mice is an interleukin-4-dependent disease with a Th2 phenotype. Am J Pathol. 2001; 159:193-203.
13. Cihakova D, Barin JG, Afanasyeva M, Kimura M, Fairweather D, Berg M, Talor MV, Baldeviano GC, Frisancho S, Gabrielson K, Bedja D, Rose NR. Interleukin-13 protects against experimental autoimmune myocarditis by regulating macrophage differentiation. Am J Pathol. 2008; 172:1195-1208.

14. Van Dyken SJ, Locksley RM. Interleukin-4- and interleukin13-mediated alternatively activated macrophages: roles in homeostasis and disease. Annu Rev Immunol. 2013; 31:317-343.

15. Locati M, Mantovani A, Sica A. Macrophage activation and polarization as an adaptive component of innate immunity. Adv Immunol. 2013; 120:163-184.

16. Martinez FO, Helming L, Gordon S. Alternative activation of macrophages: an immunologic functional perspective. Annu Rev Immunol. 2009; 27:451-483.

17. Xiao X, Gittes GK. Concise Review: New Insights Into the Role of Macrophages in beta-Cell Proliferation. Stem cells translational medicine. 2015; 4:655-658.

18. Cui J, Xu W, Chen J, Li H, Dai L, Frank JA, Peng S, Wang S, Chen G. M2 polarization of macrophages facilitates arsenic-induced cell transformation of lung epithelial cells. Oncotarget. 2017; 8:21398-21409. https:// doi.org/10.18632/oncotarget.15232.

19. Zhang L, Xu Y, Sun J, Chen W, Zhao L, Ma C, Wang Q, Sun J, Huang B, Zhang Y, Li X, Qu X. M2-like tumorassociated macrophages drive vasculogenic mimicry through amplification of IL-6 expression in glioma cells. Oncotarget. 2017; 8:819-832. https://doi.org/10.18632/ oncotarget. 13661.

20. Carroll MJ, Kapur A, Felder M, Patankar MS, Kreeger PK. M2 macrophages induce ovarian cancer cell proliferation via a heparin binding epidermal growth factor/ matrix metalloproteinase 9 intercellular feedback loop. Oncotarget. 2016; 7:86608-86620. https://doi.org/10.18632/ oncotarget.13474.

21. Lindstrom A, Midtbo K, Arnesson LG, Garvin S, Shabo I. Fusion between M2-macrophages and cancer cells results in a subpopulation of radioresistant cells with enhanced DNArepair capacity. Oncotarget. 2017; 8:51370-51386. https:// doi.org/10.18632/oncotarget.17986.

22. Rehermann B. Mature peritoneal macrophages take an avascular route into the injured liver and promote tissue repair. Hepatology. 2017; 65:376-379.

23. Barreiro O, Cibrian D, Clemente C, Alvarez D, Moreno V, Valiente I, Bernad A, Vestweber D, Arroyo AG, Martin P, von Andrian UH, Sanchez Madrid F. Pivotal role for skin transendothelial radio-resistant anti-inflammatory macrophages in tissue repair. eLife. 2016; 5.

24. Wang J, Kubes P. A Reservoir of Mature Cavity Macrophages that Can Rapidly Invade Visceral Organs to Affect Tissue Repair. Cell. 2016; 165:668-678.

25. Xiao X, Gaffar I, Guo P, Wiersch J, Fischbach S, Peirish L, Song Z, El-Gohary Y, Prasadan K, Shiota C, Gittes GK. 
M2 macrophages promote beta-cell proliferation by upregulation of SMAD7. Proc Natl Acad Sci USA. 2014; 111:E1211-1220.

26. Wang X, Lin WJ, Izumi K, Jiang Q, Lai KP, Xu D, Fang LY, Lu T, Li L, Xia S, Chang C. Increased infiltrated macrophages in benign prostatic hyperplasia $(\mathrm{BPH})$ : role of stromal androgen receptor in macrophage-induced prostate stromal cell proliferation. J Biol Chem. 2012; 287:18376-18385.

27. Spera JM, Herrmann CK, Roset MS, Comerci DJ, Ugalde JE. A Brucella virulence factor targets macrophages to trigger B-cell proliferation. J Biol Chem. 2013; 288:20208-20216.

28. Luo Y, Pollard JW, Casadevall A. Fcgamma receptor crosslinking stimulates cell proliferation of macrophages via the ERK pathway. J Biol Chem. 2010; 285:4232-4242.

29. Mutsaers SE, Whitaker D, Papadimitriou JM. Stimulation of mesothelial cell proliferation by exudate macrophages enhances serosal wound healing in a murine model. Am J Pathol. 2002; 160:681-692.

30. Lin S, Qiu M, Chen J. IL-4 Modulates Macrophage Polarization in Ankylosing Spondylitis. Cell Physiol Biochem. 2015; 35:2213-2222.

31. Pan B, Liu G, Jiang Z, Zheng D. Regulation of renal fibrosis by macrophage polarization. Cell Physiol Biochem. 2015; 35:1062-1069.

32. Sica A, Mantovani A. Macrophage plasticity and polarization: in vivo veritas. J Clin Invest. 2012; 122:787-795.
33. Li Z, Guo C, Liu X, Zhou C, Zhu F, Wang X, Wang Q, Shi Y, Wang J, Zhao W, Zhang L. TIPE2 suppresses angiogenesis and non-small cell lung cancer (NSCLC) invasiveness via inhibiting Rac1 activation and VEGF expression. Oncotarget. 2016; 7:62224-62239. https://doi. org/10.18632/oncotarget.11406.

34. Li F, Zhu X, Yang Y, Huang L, Xu J. TIPE2 Alleviates Systemic Lupus Erythematosus Through Regulating Macrophage Polarization. Cell Physiol Biochem. 2016; 38:330-339.

35. Lou Y, Liu S, Zhang C, Zhang G, Li J, Ni M, An G, Dong M, Liu X, Zhu F, Zhang W, Gao F, Chen YH, et al. Enhanced atherosclerosis in TIPE2-deficient mice is associated with increased macrophage responses to oxidized low-density lipoprotein. J Immunol. 2013; 191:4849-4857.

36. Sun H, Gong S, Carmody RJ, Hilliard A, Li L, Sun J, Kong L, Xu L, Hilliard B, Hu S, Shen H, Yang X, Chen YH. TIPE2, a negative regulator of innate and adaptive immunity that maintains immune homeostasis. Cell. 2008; 133:415-426.

37. Weischenfeldt J, Porse B. Bone Marrow-Derived Macrophages (BMM): Isolation and Applications. CSH protocols. 2008; 2008:pdb prot5080. 